\title{
Change or Perish
}

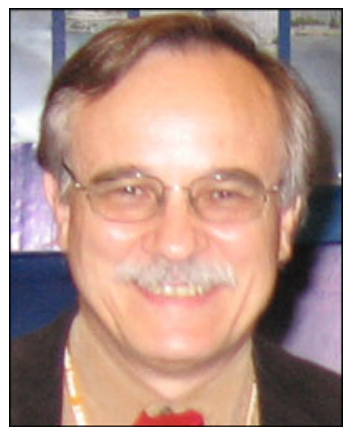

The title of this editorial comes from the 2005 Pritzker Prize winner's speech. Thom Mayne is the principal at Morphosis, an architecture firm in Santa Monica California. With regard to computational advancement of ideas to reality he says: "The tools we now utilize simplify these potentialities and make them logical, allowing us to produce spaces that even ten years ago would have been difficult to conceive, much less build. Anything that is possible is realizable." Similar thoughts must surely permeate forefront design teams in automotive, aerospace, and other engineering industries. Some cutting edge entertainment and retail companies seem to have had related epiphanies.

In materials discovery there are also examples of computational adaptations needed for progress (or survival) and, similar to the industries listed above, commercial advantages are a strong driver. CALPHAD is an example well known to our community, while bio-tech companies represent a dynamic new example where ideas and drug design are developed in silico (an appropriate and common biology way of saying 'in computers').

There are certainly commercial pressures pushing for the development of new and better materials. For example, in the news this past year are articles calling for the development of stronger non-rare earth permanent magnets as China has gained a near monopoly in the mining and production of rare earth products. With their push for wind energy, China will soon be able to consume all their rare earth production for domestic wind turbine installations. There are calls for stronger materials, harder materials, higher temperature superconductors, materials that can better withstand nuclear reactor radiation, etc. But unlike some of the examples above, computer aided bulk material discovery is not yet a routine undertaking. The underlying quantum processes can be complex (beyond the purview of standard electronic structure algorithms), the number of possible combinatorical atomic configurations can be prohibitive to evaluate with precision, and the necessity of structural stability at elevated temperatures can hinder computational evaluations.

Government sponsored research in the early stages can nurture the development of computational methods, which in turn can lead to commercial development and promote greater applications. Recent statistics show that Europe has surpassed the US in the number of publications dealing with computational materials science and chemistry by $65 \%$, and China's publications in this area are projected to surpass those of the US by 2012. Many software teams in Europe are centered around suites of codes, which are available for downloading or for sale, although the progenitors of most of the codes were developed in the US. A list of 24 such codes is presented in the recent publication "Numerical Methods for Electronic Structure Calculations of Materials" by Y. Saad, J. R. Chelikowsky, and S. M. Shontz (SIAM Rev., Vol 52 (No. 1), 2010, p 3-54). There are a number of other codes that could have been listed, such as GAMESS (with 150,000 users). Bill Gates pioneered the concept that progress (and profit) is in the software, not the hardware. That may prove to be the case for advancing materials development as well.

A bright spot is the establishment at the DOE National Nano-Centers of integrated theory/computational groups. Also, I recently attended a "Computational Scattering" workshop, which was held to promote the greater use of modern computational tools at the large x-ray synchrotron and neutron scattering facilities. A notable development is the EU sponsored European Theoretical Spectroscopy Facility, where software and computational resources are created and directed to assist experimentalists, commercial enterprises and governments. As at major experimental facilities, users can propose projects to be undertaken by various "beamlines". (see http://www.etsf.eu/) With computing capability continuing to dramatically increase (toward the exascale ${ }^{\dagger}$ ), successful materials discovery in silico should be a success story accelerating for at least the next 50 years.

\author{
Bruce Harmon \\ Associate Editor \\ Journal of Phase Equilibria and Diffusion
}

\footnotetext{
${ }^{\dagger}$ See the author's previous editorial in the August 2009 issue of this journal.
} 